\title{
Editorial
}

\section{Editorial: Applied Mechanics}

\author{
Magd Abdel Wahab +(i) \\ Soete Laboratory, Ghent University, Technologiepark Zwijnaarde 46, B-9052 Zwijnaarde, Belgium; \\ magd.abdelwahab@ugent.be \\ + Editor-in-Chief Applied Mechanics.
}

Received: 28 October 2019; Accepted: 1 November 2019; Published: 6 November 2019

Mechanics is a branch of physics that describes the theoretical aspects related to the response of objects to external forces and displacements. It can be divided into three main broad categories, namely, classical mechanics, fluid mechanics, and quantum mechanics. Classical mechanics and fluid mechanics, respectively, concentrate on the study of mechanics of solids and fluid at the macroscopic scale, whereas quantum mechanics concentrates on the study of physics of objects at the atomic scale. The journal Applied Mechanics is dedicated to topics related to classical mechanics and fluid mechanics theories and applications.

Classical mechanics is divided into two main categories, namely, statics and dynamics. Statics concentrates on studies and analyses of bodies at rest, whereas dynamics concentrates on studies and analyses of bodies in motion. Furthermore, statics analysis can be divided into two types, namely, statics of rigid bodies and statics of deformable bodies. Statics of rigid bodies studies and analyzes forces acting on nondeformable or rigid structures. In contrast, statics of deformable bodies is concerned with the analysis of deformable structures and is related to topics on structural mechanics and strength of materials. Moreover, dynamics can be divided into two branches, i.e., kinematics and kinetics. In kinematics, bodies in motion are analyzed using time, displacements, velocities, and accelerations, without reference to forces and masses, whereas in kinetics, the effects of forces and masses are considered and analyzed.

Opposite to solids, which resist external forces by elastic deformation, fluids continually flow and deform under applied shear loads. Fluids can be either liquids or gases, which may behave similarly when there are no surface effects. Similar to classical mechanics, fluid mechanics can be divided into two main categories, i.e., statics and dynamics. Fluid statics, also known as hydrostatics, is concerned with the study of incompressible fluid at rest, while fluid dynamics is concerned with the study of fluids in motion and describes the flow of fluids.

Applied mechanics is the practical application of the theories of classical mechanics and fluid mechanics to general engineering problems. It is also sometimes termed as engineering mechanics because it has applications to a wide range of engineering disciplines, e.g., civil, mechanical, aerospace, materials, and biomedical engineering. In civil engineering, applied mechanics has many applications including structural engineering, structural design, coastal engineering, geotechnical engineering, construction engineering, and earthquake engineering. In mechanical engineering, applied mechanics is used in machine elements, mechatronics and robotics, structural analysis, design and drafting, friction stir welding, acoustical engineering, and nanotechnology. The use of applied mechanics in aerospace engineering includes aerospace structural mechanics, aircraft design, aerospace propulsion, aerodynamics, and flight mechanics.

In Figure 1, a flowchart is presented to illustrate the classification of classical mechanics and fluid mechanics, as well as their practical applications to different engineering disciplines. These topics are the focus of the journal Applied Mechanics. This journal will adopt an open access model, and all manuscripts will be rapidly processed and undergo rigorous peer review. We are committed to presenting the latest research results in the field, as well as to share them within the research community 
without any barriers. We also hope that the authors will bring new advances from various fields of applied mechanics.

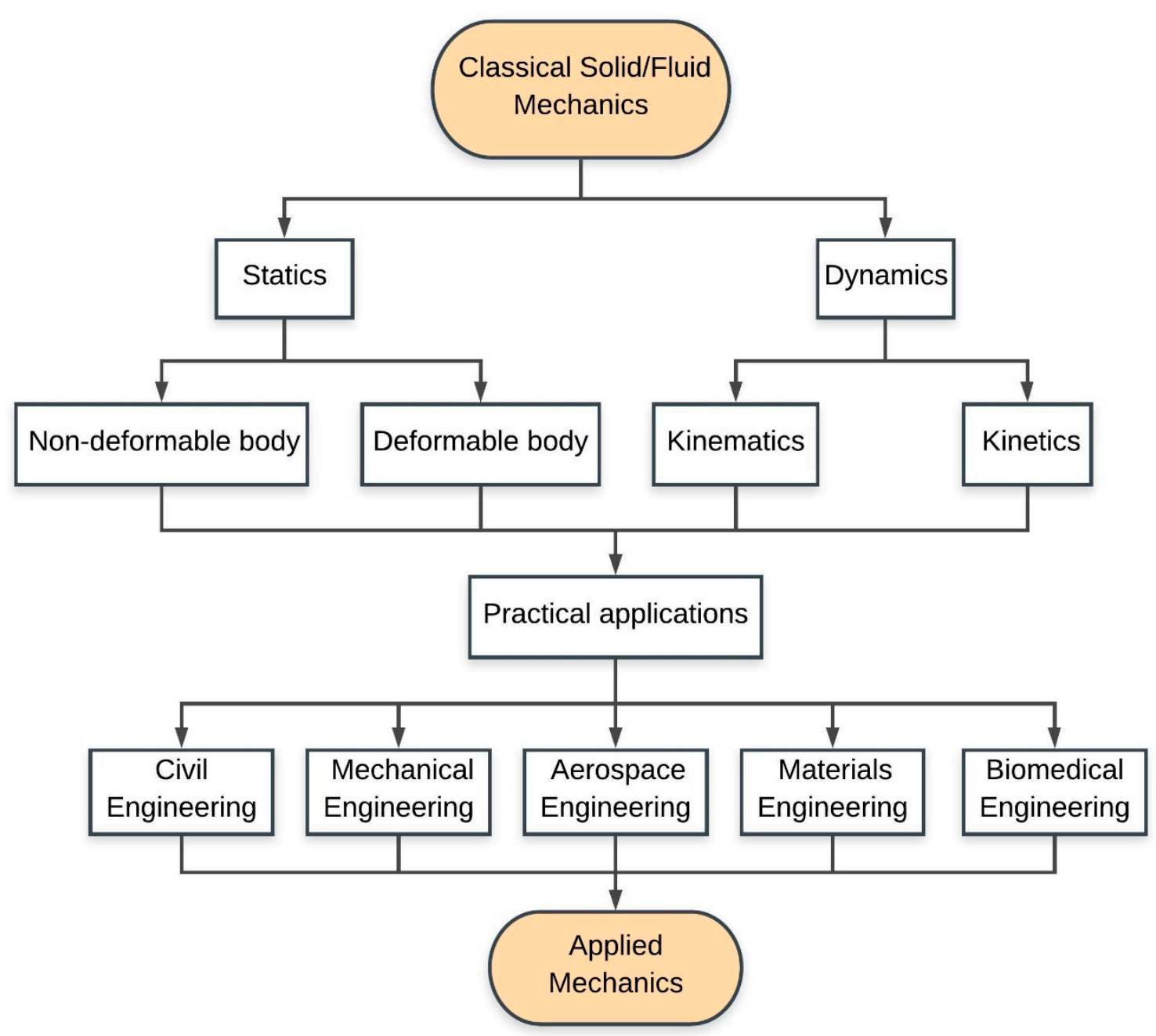

Figure 1. Flowchart of Applied Mechanics.

(C) 2019 by the author. Licensee MDPI, Basel, Switzerland. This article is an open access article distributed under the terms and conditions of the Creative Commons Attribution (CC BY) license (http://creativecommons.org/licenses/by/4.0/). 